\title{
Induction of the cytokine signal regulator SOCS3/CIS3 as a therapeutic strategy for treating inflammatory arthritis

\author{
Takanori Shouda, ${ }^{1,2}$ Takafumi Yoshida, ${ }^{1,3}$ Toshikatsu Hanada, ${ }^{1}$ Toru Wakioka, ${ }^{1,4}$ \\ Masanobu Oishi, ${ }^{1}$ Kanta Miyoshi, ${ }^{1,4}$ Setsuro Komiya, ${ }^{4}$ Ken-ichiro Kosai, ${ }^{5}$ \\ Yasushi Hanakawa, ${ }^{6}$ Koji Hashimoto, ${ }^{6}$ Kensei Nagata, ${ }^{2}$ and Akihiko Yoshimura ${ }^{1}$
}

${ }^{1}$ Division of Molecular and Cellular Immunology, Medical Institute of Bioregulation, Kyushu University, Fukuoka, Japan
${ }^{2}$ Department of Orthopedic Surgery, and
${ }^{3}$ Second Department of Internal Medicine, Faculty of Medicine, Kurume University, Kurume, Japan
${ }^{4}$ Department of Orthopedic Surgery, Faculty of Medicine, Kagoshima University, Kagoshima, Japan
${ }^{5}$ Department of Regeneration of the Cardiovascular System, Gifu University School of Medicine, Gifu, Japan
${ }^{6}$ Department of Dermatology, Ehime University Medical School, Shitukawa, Japan

Address correspondence to: Akihiko Yoshimura, Division of Molecular and Cellular Immunology,

Medical Institute of Bioregulation, Kyushu University, Maidashi, Higashi-ku, Fukuoka 812-8582, Japan.

Phone: 81-92-642-6823; Fax: 81-92-642-6825; E-mail: yakihiko@bioreg.kyushu-u.ac.jp.

Received for publication June 19, 2001, and accepted in revised form October 22, 2001.

\begin{abstract}
Immune and inflammatory systems are controlled by multiple cytokines, including ILs and INFs. These cytokines exert their biological functions through Janus tyrosine kinases and STAT transcription factors. One such cytokine, IL-6, has been proposed to contribute to the development of rheumatoid arthritis (RA). We found that STAT3 was strongly tyrosine phosphorylated in synovial tissue of RA patients, but not those with osteoarthritis. Blockade of the IL-6-gp130-JAK-STAT3-signaling pathway might therefore be beneficial in the treatment of RA. We show here that the mRNA for the endogenous cytokine signaling repressor CIS3/SOCS3 is abundantly expressed in RA patients. To determine whether CIS3 is effective in treating experimental arthritis, a recombinant adenovirus carrying the CIS3 cDNA was injected periarticularly into the ankle joints of mice with antigeninduced arthritis or collagen-induced arthritis (CIA). Periarticular injection of CIS3 adenovirus drastically reduced the severity of arthritis and joint swelling compared with control groups. CIS3 was more effective than a dominant-negative form of STAT3 in the CIA model. Thus, induction of CIS 3 could represent a new approach for effective treatment of RA.
\end{abstract}

J. Clin. Invest. 108:1781-1788 (2001). DOI:10.1172/JCI200113568.

\section{Introduction}

Rheumatoid arthritis (RA) is an autoimmune disease characterized by chronic inflammation of multiple joints, resulting in mononuclear cell infiltration, development of pannus, progressive cartilage destruction, and bone erosion. Although the cause of RA remains unknown, it has been suggested that cytokines, especially proinflammatory cytokines such as TNF- $\alpha$, IL-1, and IL- 6 derived from activated synovial cells, play a pivotal role in the pathology of the disease $(1,2)$. Among these cytokines, TNF- $\alpha$ has been the most extensively investigated as a target in the efforts to treat RA. Anti-TNF- $\alpha$ mAbs markedly ameliorate joint involvement in the majority of patients with RA $(3,4)$. It is well known that IL-6 is one major target gene of TNF- $\alpha$. IL- 6 has been proposed to contribute to the development of arthritis; IL- 6 induces proliferation of synovial fibroblastic cells (5) and formation of osteoclasts in association with soluble IL-6 receptors (6). Involvement of IL-6 in RA is also suggested by recent reports of IL- 6 gene-disrupted mice that were resistant to antigen-induced arthritis (AIA)
$(7,8)$ and collagen-induced arthritis (CIA) (9). Therefore, IL- 6 could be another therapeutic target of RA. Recently, anti-IL- 6 receptor Ab's have undergone successful trials as a therapy for RA patients (10).

IL-6 and its related cytokines, including leukemia inhibitory factor (LIF), oncostatin M (OSM), and IL-11, preferentially activate JAK tyrosine kinases and the STAT3 transcription factor. STAT3 activation was found exclusively in synovial tissue from RA, but not osteoarthritis (OA), patients. Therefore, elucidation of the regulatory mechanisms of the JAK-STAT3 pathway is important in understanding the inflammation associated with RA. Recently, we cloned JAB/SOCS1/SSI1 and CIS3/SOCS3/SSI3, which inhibit JAK tyrosine kinase activity (11). Many reports have indicated that CIS3 is induced not only by various inflammatory and antiinflammatory cytokines such as IL-6, IFN- $\gamma$, IL-10, but also by LPS, and that CIS3 negatively regulates STAT functions $(12,13)$. CIS3 has been shown to interact with gp130 and JAKs, and this interaction results in relatively specific inhibition of gp130 signaling (14-16). Moreover, CIS3 also inhibits LPS-induced NO synthesis in mono- 
cytic cells, although the mechanism has not been clarified (13). However, these studies used cultured primary cells or cell lines, and few focused on the relationship between CIS3 and disease. Recently, we demonstrated that CIS3 plays a negative regulatory role in STAT3 activation and intestinal inflammation (17). Here, we also demonstrate hyperactivation of STAT3 and CIS3 expression in RA, as well as in murine RA models.

The goal of RA treatment is to inhibit synovial tissue hyperplasia, because it forms the pannus tissue that irreversibly destroys cartilage and bone of the affected joint. STAT3 has been shown to play an important role in cell proliferation and antiapoptosis (18). Hyperactivation of STAT3 is probably involved in proliferation and/or antiapoptosis of RA synoviocytes (5-7). Since CIS3 is strongly induced by IL- 6 , as well as TNF- $\alpha$, CIS3 may play a negative regulatory role in the progression of RA. Therefore, we examined the efficacy of adenovirus-delivered dominant-negative (dn) STAT3 (18) and CIS3 in reducing inflammation in a mouse RA model. Our results showed that adenoviral gene delivery of the CIS3 and dnSTAT3 genes into the synovial tissues of mice with AIA and CIA significantly suppressed pathological changes associated with arthritis. We also found that CIS3 was more effective than dnSTAT3 in the CIA model. Given the selective expression of the CIS3 gene in synovial cells, induction of the CIS3 protein levels could represent an effective new therapeutic strategy for the treatment of RA.

\section{Methods}

Synovial tissues and isolation of synoviocytes. Synovial samples were obtained from three RA patients during total joint replacement surgery or synovectomy at the Kurume University Medical Center. All patients fulfilled the American College of Rheumatology criteria for RA (19). Synovial tissues were also collected from patients with OA. These samples were collected with the informed consent of the patients. To isolate synoviocytes, the collected synovial tissues were digested with $5 \mu \mathrm{g} / \mathrm{ml}$ collagenase (Sigma Chemical Co., St. Louis, Missouri, USA) and $1.5 \mu \mathrm{g} / \mathrm{ml}$ DNase (Sigma Chemical Co.), and were passed through a wire mesh to prepare isolated cells. Cells $\left(5 \times 10^{5}\right)$ were placed in 10 -cm dishes with $10 \mathrm{ml} \mathrm{RPMI}$ supplemented with $10 \%$ FCS, $2 \mathrm{mM}$ L-glutamine, $50 \mathrm{U} / \mathrm{ml}$ penicillin, and 50 $\mu \mathrm{g} / \mathrm{ml}$ streptomycin. At $80 \%$ confluence, cells were removed by trypsinization and split for subsequent culture passages. Passage 3-7 cells were used and considered a homogeneous population of fibroblasts.

Recombinant adenovirus. Adenoviral vectors containing the genes for LacZ (AxCALacZ), Myc-tagged CIS3 (AxCACIS3) and HA-tagged dnSTAT3 (AxCAdnSTAT3) (18), which contain a CAG promoter (chicken $\beta$-actin promoter with cytomegalovirus enhancer), were prepared by homologous recombination in 293 cells as described previously (20). A recombinant adenovirus with the bacterial LacZ gene driven by the same promoter, AxCALacZ, was provided by I. Saito of the University of Tokyo. The viral preparations were titrated with a plaque-forming assay on 293 cells. The number of virus particles (measured in PFU) per cell was expressed as moi. For in vivo gene transfer, $50 \mu \mathrm{l}$ of $2 \times 10^{9} \mathrm{PFU} / \mathrm{ml}$ recombinant viruses in saline were injected periarticularly to ankle joints and wrist joints.

Cytokine production and proliferation assays. Infection of cultured synoviocytes with adenovirus vectors was performed following the method described previously $(20-22)$. Briefly, the cells in culture were incubated with adenovirus $\left(10^{10} \mathrm{PFU} / \mathrm{ml}\right)$ diluted with DMEM for 1 hour at $37^{\circ} \mathrm{C}$, after which $10 \mathrm{vol}$ of medium containing $10 \%$ FBS were added. After infection, cells were removed by trypsinization and were seeded into 24-well plates at a concentration of $5 \times 10^{4}$ cells/well in $1 \mathrm{ml}$ of RPMI containing 10\% FCS. Conditioned medium (CM) was removed at various time points and frozen at $-20^{\circ} \mathrm{C}$ until assayed by ELISA. Quantification of IL-1 $\beta$, TNF- $\alpha$, and IL- 6 was performed using kits from Biosource International (Camarillo, California, USA). Cell proliferation assays were performed as described previously $(21,22)$. AIA and CIA models and adenovirus infection of mice. Male C57BL/ 6 mice and DBA/1J mice were purchased from SLC Japan Co. (Shizuoka, Japan). For AIA, C57BL/6 mice were immunized intradermally at the base of the tail and four footpads with $100 \mu \mathrm{g}$ of methylated BSA (mBSA; Sigma Chemical Co.) emulsified with an equal volume of complete Freund's adjuvant (Difco Laboratories, Detroit, Michigan, USA) as described $(8,22)$. They were injected with $2 \times 10^{9}$ Bordetella pertussis organisms (Kaken Pharmaceutical, Tokyo, Japan). This procedure was repeated on day 7 . On day $21,100 \mu \mathrm{g}$ mBSA in $10 \mathrm{ml}$ of saline was injected into bilateral ankle joints. Adenovirus was injected into ankle joints on day 6. CIA was induced by established methods as described previously $(23,24)$. Briefly, DBA/1J mice were immunized by intradermal injection at the base of the tail with $100 \mu \mathrm{g}$ of bovine type II collagen (Cosmo Bio Co., Tokyo, Japan) in $0.05 \mathrm{M}$ acetic acid, emulsified with an equal volume of Freund's complete adjuvant. On day 21, mice received a booster dose of intradermal injection and an equal volume of incomplete Freund's adjuvant. To synchronize the onset of arthritis, $40 \mu \mathrm{g}$ LPS was injected intraperitoneally on day 28. Adenovirus was injected into ankle joints and wrists on day 28 or on day 32 . All procedures involving animals were performed in accordance with the guidelines of the Animal Experimentation Ethics Committee of our institutions.

Clinical assessment of arthritis. CIA development was inspected every other day, and inflammation of the four paws was graded from 0 to 4 : grade 0 , paws with no swelling and focal redness; grade 1, paws with swelling of finger joints; grade 2, paws with mild swelling of ankle or wrist joints; grade 3, paws with severe inflammation of the entire paws; and grade 4, paws with deformity or ankylosis. Each paw was graded and the four scores were totaled so that the maximum possible score per mouse was 16 .

Histological examination and immunohistochemistry. The legs and hind paws of mice were removed, fixed with $4 \%$ 
paraformaldehyde in PBS, and then decalcified for 10 days with EDTA and embedded in paraffin. The paraffin sections were stained with hematoxylin-eosin and Safranin O-fast green. The slides were evaluated histologically by two independent observers, and the gradation of arthritis was scored from 0 to 4 according to the intensity of lining layer hyperplasia, mononuclear cell infiltration, and pannus formation, as described previously (23): 0 , normal ankle joint; 1 , normal synovium with occasional mononuclear cells; 2 , definite arthritis, a few layers of flat to rounded synovial lining cells and scattered mononuclear cells; 3 , clear hyperplasia of the synovium with three or more layers of loosely arranged lining cells and dense infiltration with mononuclear cells; 4 , severe synovitis with pannus and erosions of articular cartilage and subchondral bone.

For LacZ staining, LacZ adenovirus-infected tissue was fixed with $4 \%$ formalin and incubated overnight at $37^{\circ} \mathrm{C}$ in X-gal solution [5 $\mathrm{mM} \mathrm{K} 4 \mathrm{Fe}(\mathrm{CN}) 6 ; 5 \mathrm{mM} \mathrm{K} 3 \mathrm{Fe}(\mathrm{CN}) 6$; $2 \mathrm{mM} \mathrm{MgCl} 2$; and $2 \mathrm{mM}$ 5-bromo-4-chloro-3-indolyl- $\beta$ galactoside in PBS] as described (21).

Immunoblot, Northern bybridization, and RT-PCR analysis. Synovial tissues were isolated from hind paws by removing the skin, muscle, fatty tissues, bone, and tendons, then immediately frozen in liquid nitrogen and stored at $-80^{\circ} \mathrm{C}$. The whole joint tissues of mice and specimens of human patients without bone were homogenized in 2-4 $\mathrm{ml}$ of a lysis buffer containing 50 mM Tris- $\mathrm{HCl}$ (pH 8.0) 0.5\% NP-40, 1 mM EDTA, 150 $\mathrm{mM} \mathrm{NaCl}, 10 \%$ glycerol, $1 \mathrm{mM}$ sodium vanadate, 50 $\mathrm{mM}$ sodium fluoride, $10 \mathrm{mM}$ sodium pyrophosphate, and $1 \mathrm{mM}$ PMSF with a protease inhibitor cocktail (Sigma Chemical Co.) (17). The extracts were cleared by spinning at $10,000 \mathrm{~g}$ at $4^{\circ} \mathrm{C}$ for 15 minutes and then diluted with the lysis buffer to achieve about $2 \mathrm{mg} / \mathrm{ml}$ protein concentration. The total cell extracts were resolved by SDS-PAGE, and proteins were detected by immunoblotting as described (17). Anti-phosphoSTAT3-specific Ab's were purchased from New England BioLabs Inc. (Beverly, Massachusetts, USA) and Upstate Biotechnology Inc. (Lake Placid, New York, USA).

Total RNA from various kinds of tissue was prepared with TRIzol (Life Technologies Inc., Grand Island, New York, USA) according to the manufacturer's instructions. Total RNA $(5 \mu \mathrm{g})$ was separated on $1 \%$ agarose gels containing $2.4 \%$ formaldehyde and then transferred to positively charged nylon membranes. After fixation under calibrated ultraviolet irradiation, the membranes were hybridized with digoxigenin-labeled (DIG-labeled) riboprobes and visualized using alkaline phosphataselabeled anti-DIG Ab according to the manufacturer's instructions (Roche Molecular Biochemicals Inc., Mannheim, Germany). The probe cDNA for CIS3 and GAPDH has been described previously (17). For RTPCR, primers for murine IL-1 $\beta$, IL-6, TNF- $\alpha$, and IL-10 were described previously $(17,25)$ detected by standard RT-PCR methods using Standard GeneAmp RNA PCR kit (PE Biosystems, Foster City, California, USA) according to the manufacturer's instructions.
Statistical methods. The significance of difference between group means was determined by a MannWhitney $U$ test in the program SigmaStat (SPSS Software Products, Chicago, Illinois, USA).

\section{Results}

Activation of STAT3 and induction of CIS 3 in RA patients and mouse RA models. We have shown that STAT3 is correlated with the severity of intestinal inflammation (17). First, we compared STAT3 activation in RA and OA patients using immunohistochemistry with anti-phospho-STAT3-specific Ab's. As shown in Figure 1a, activated STAT3 was detected in the nucleus of RA synovial cells, but not in OA samples. This suggests that STAT3 activation is related to hyperplasia of synovial tissues. We have also demonstrated that cytokine signal suppressor CIS3 is induced in colitis (17). We therefore examined CIS3 expression in RA and OA patients by Northern blot analysis. As shown in Figure 1b, CIS3
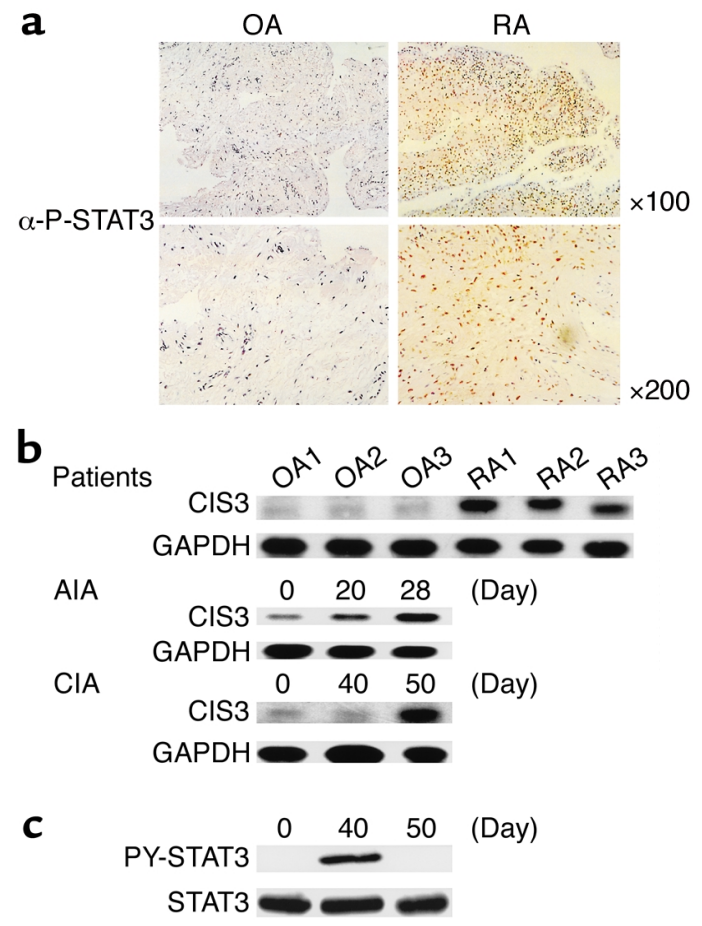

\section{Figure 1}

CIS3 and STAT3 activation in synovial tissue from patients with RA and OA. (a) Immunohistochemical detection of activated STAT3 in synovial tissues. Slides with sectioned tissues of 7-mm thickness were immunostained with anti-phospho-STAT3-specific Ab's ( $\alpha$-P-STAT3) and were lightly stained with hematoxylin. (b) Expression of CIS3 mRNA in RA or OA patients and in the mouse AIA or CIA model. Total RNA was extracted from three OA and RA patients' synovial tissues or the ankle joints of mice treated with mBSA (AIA) or bovine type II collagen (CIA). For AIA, mBSA was injected on days 0,7 , and 21. For CIA, type II collagen was injected on days 0 and 21, and RNA was extracted on indicated days. Northern hybridization was performed with cDNA probes for coding regions of human and mouse CIS3 and with control probe. (c) Cell extracts from the same CIA samples were immunoblotted with anti-phosphorylated STAT3 (PY-STAT3) or anti-STAT3 Ab's (STAT3). 


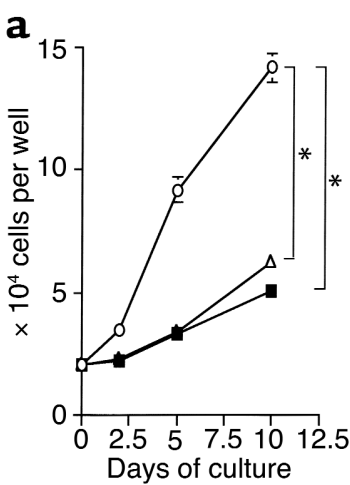

b

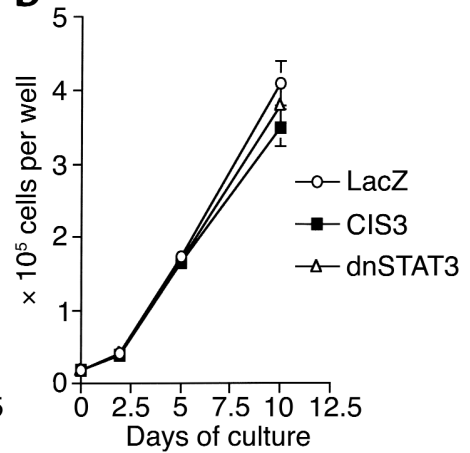

C
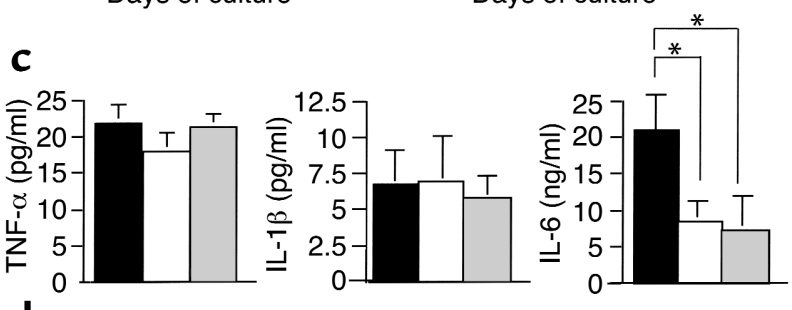

d

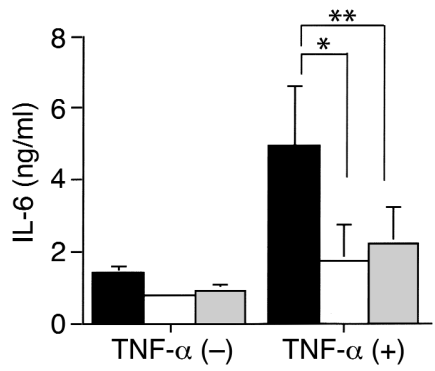

Figure 2

Effects of dnSTAT3 and CIS3 overexpression on proliferation and cytokine secretion of RA synovial fibroblasts. ( $\mathbf{a}$ and $\mathbf{b}$ ) Cell growth curves of rheumatoid synoviocytes and $\mathrm{NIH}-3 \mathrm{~T} 3$ cells inoculated with LacZ, dnSTAT3, or CIS3 adenovirus (moi $=50$ ). Data represent mean \pm SD of triplicate measurements. (c and d) Rheumatoid synoviocytes inoculated with LacZ, CIS3, or dnSTAT3 virus were cultured for 24 hours in the presence of $10 \%$ FCS (c) or in the absence $(-)$ or presence $(+)$ of $10 \mathrm{ng} / \mathrm{ml} \mathrm{TNF-} \alpha$ in $0.1 \%$ FCS (d). Then, TNF- $\alpha$, IL-1 $\beta$, and IL- 6 concentrations in conditioned medium were detected by ELISA. Data are expressed as mean \pm SD derived from three separate experiments. ${ }^{*} P<0.01 \mathrm{vs.} \mathrm{LacZ,}{ }^{*} P<0.05$ vs. LacZ.

was expressed strongly in synovial tissues from RA patients, but only weakly in OA synovial tissues.

We also examined CIS3 expression using the mouse AIA and CIA models. AIA is an animal model of arthritis initially induced in rabbits and extended into mice (23). Although AIA is a model of monoarthritis, it has the advantage of precise and fair histological comparisons among individual mice. Mice were immunized with mBSA on days 0,7 , and 20 , and the joint inflammation became severe at day 28. CIS3 mRNA was observed at low levels in normal joint tissues. However, CIS3 mRNA levels increased as arthritis developed (Figure 1b). CIA is a well-established animal model of RA in which the disease is elicited by immunization of genetically susceptible mice with type II collagen, and it bears many of the histological features and both cellular and humoral immune responses characteristically found in
RA (24). The joint inflammation became severe at day 40 (see Figure 5a and Figure 6), and STAT3 phosphorylation was evident at day 40 but decreased at day 50 (Figure 1c) whereas CIS3 mRNA levels increased drastically at day 50 (Figure 1b). Thus, there seems to be a time lag between inflammation/STAT3 activation and CIS3 expression. This is consistent with our idea that CIS3 function as a negative feedback regulator of STAT3.

CIS3 gene transfer decreases proliferation and IL-6 levels in $R A$ synovial fibroblasts. To elucidate the significance of STAT3 activation and CIS3 induction in RA, we overexpressed dnSTAT3 and CIS3 in RA synoviocytes isolated from patients, using adenoviral gene transfer. We infected cultured synovial fibroblasts in vitro with adenovirus carrying CIS3 (AxCACIS3), dnSTAT3 (AxCAdnSTAT3), or LacZ (AxCALacZ). Adenovirus was inoculated at moi $=50$, and $100 \%$ of cells were infected as assessed by LacZ staining (data not shown). AxCACIS3 and AxCAdnSTAT3 but not AxCALacZ infection strongly suppressed proliferation of synovial fibroblasts in 10\% FCS (Figure 2a). Thus, proliferation of synovial fibroblasts strictly depends on JAK/STAT3 pathway. Suppression of proliferation by CIS3 and dnSTAT3 was not observed in NIH-3T3 fibroblasts (Figure 2b), suggesting that STAT3-dependent growth is a specific feature of synovial fibroblasts.

We next investigated the effect of CIS3 and dnSTAT3 on secretion of proinflammatory cytokines. The levels of TNF- $\alpha$, IL- $1 \beta$, and IL- 6 were analyzed in the conditioned medium (CM) of AxCACIS3-, AxCAdnSTAT3-, and AxCALacZ-infected RA synovial fibroblasts (Figure 2 , c and d). Serum-induced as well as TNF $\alpha$-induced IL-6 production was significantly decreased by infection with AxCACIS3 and AxCAdnSTAT3, but not with AxCALacZ (controls). However, TNF- $\alpha$ and IL- $1 \beta$ levels were not altered by infection with AxCACIS3 and AxCAdnSTAT3 (Figure 2c). These data suggest that JAK/STAT pathway is involved in IL-6 production in RA synovial fibroblasts, and that CIS3 and dnSTAT3 adenovirus can suppress not only IL-6 signaling but also a part of TNF- $\alpha$ signaling.

CIS3 gene therapy suppresses AIA. We attempted to suppress AIA using adenovirus carrying CIS3 or dnSTAT3. It has been shown that intra-articular or periarticular injection of adenovirus results in efficient and high expression of exogenous genes $(21,22)$. Consistently, in situ staining for $\beta$-gal showed a diffuse blue staining in the synovial tissues and periarticular tissues of injected ankles (Figure 3a). Light microscopy revealed the transgene expression in about $60-80 \%$ of synovial cells by our experimental conditions (Figure $3 \mathrm{~b}$ ). Western blot analysis demonstrated that CIS3 protein was detected in the ankle joint 7 days after $10^{8} \mathrm{PFU}$ AxCACIS3 injection. However, after 28 days, CIS 3 expression was no longer detected (data not shown). This is consistent with previous reports of transient adenoviral gene expression $(21,22)$.

We then treated AIA mice by periarticular adenoviral transfer of CIS3 and dnSTAT3 genes. Six days after the first immunization, we injected AxCACIS3 or AxCAdn- 
a

b

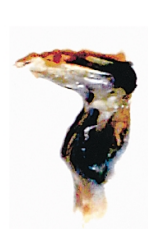

C

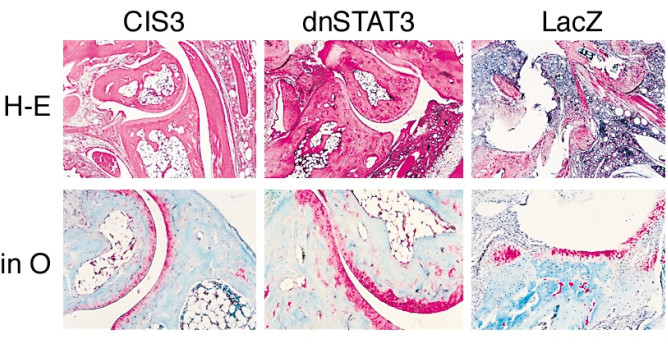

Z

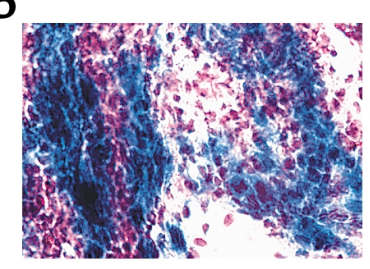

d
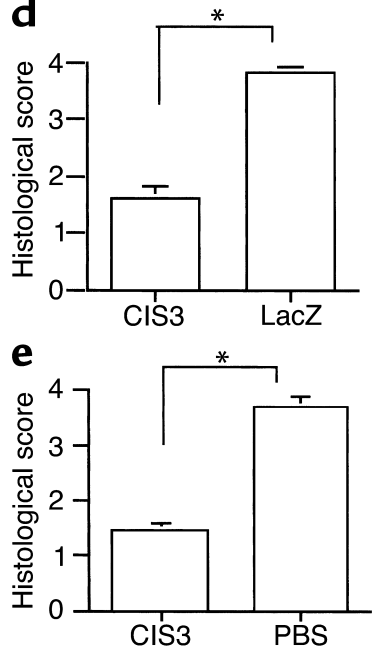

Safranin O
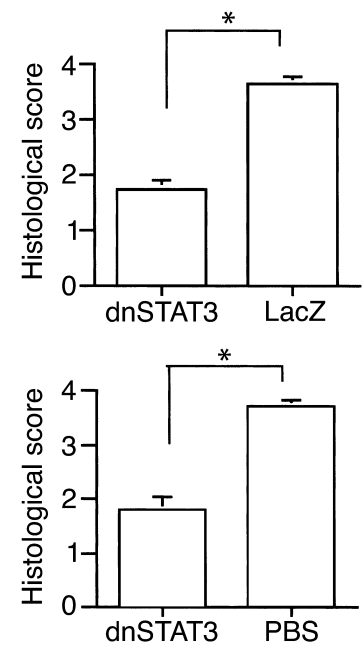

Figure 3

Effects of CIS3 and dnSTAT adenoviruses on histopathology in the AIA model. In vivo expression of transgenes by adenovirus vectors. (a) After AIA induction, $10^{8} \mathrm{PFU}$ of AxCALacZ were injected into the ankle joints. Then, 7 days later, the hind paw was removed and stained with X-gal. (b) Microscopic view of synovial cells stained with $X$-gal and hematoxylin $(\times 400)$. (c) Representative histopathologies of the ankle joints stained with hematoxylin and eosin (H-E) and Safranin-O $(\times 100)$. Adenovirus or PBS was injected into ankle joints on day 6,1 day before the second immunization. Mice were inoculated with PBS or LacZ virus and CIS3 or dnSTAT3 virus into each ankle joint. Thirty-five days after the first immunization, mice were sacrificed and subjected to histopathological examination. ( $\mathbf{d}$ and $\mathbf{e}$ ) Histological examinations of a total of 15 mice for each pair were scored from 0 to 4 as described in the Methods. ${ }^{*} P<0.01$.

STAT3 into the left ankle joints and the same amount of AxCALacZ or PBS into the right ankle joints of the same mice. Antigen (mBSA) was injected at day 7 and at day 21. The mice were sacrificed 35 days after the first immunization, and histological examination was performed. As shown in Figure 3, c-e, both CIS3 and STAT3 suppressed mononuclear cell infiltration and pannus formation. Safranin O staining revealed that cartilage was almost completely destroyed in AxCALacZ- or PBS-injected mice, while articular carti-

lage was well preserved in mice treated with CIS3- or dnSTAT3 adenovirus (Figure 3c; data for PBS was not shown). The effects of CIS 3 and dnSTAT 3 adenoviruses were comparable (Figure 3, $\mathrm{d}$ and e).

We then examined the effect of ectopic expression of CIS3 and dnSTAT3 on STAT3 activation (Figure 4a) and cytokine expression (Figure 4b) in the AIA model. Local expression of CIS3 and dnSTAT3 suppressed STAT3 activation after the induction of AIA on day 28. RT-PCR analysis confirmed strong suppression of IL- 6 production by CIS3 and dnSTAT3 overexpression (Figure $4 \mathrm{~b}$ ). However, TNF- $\alpha$, IL-1 $\beta$, and IL-10 levels were not affected in arthritic joints (Figure $4 b$ ). These cytokine expression patterns were similar to those found in synovial fibroblasts infected with CIS3 or dnSTAT3 adenoviruses (Figure 2), as well as to those found in AIA models in IL-6-deficient mice (8).

CIS3 gene therapy suppresses CIA. To evaluate further the therapeutic effect of CIS 3 and dnSTAT3 adenoviruses in RA, we applied the adenoviruses to CIA. Eight-weekold DBA/1J male mice were immunized with bovine type II collagen emulsified in complete adjuvant. After 21 days, the mice received a booster dose using the same amount of collagen in incomplete adjuvant, and disease pathology was observed commencing 28 days after immunization. To synchronize the onset of arthritis, LPS was injected intraperitoneally on day $28(26,27)$.

First, to examine the therapeutic effects of CIS3 and dnSTAT3 gene transfer in CIA, AxCACIS3 or AxCAdnSTAT3 were administered by periarticular injection into the wrist and ankle joints of mice on day 28 after the first immunization (Figure 5, a-c). The severity of arthritis (Figure 5, a-c), paw thickness, and redness (Figure $5 \mathrm{~b}$ ) were all markedly reduced in the AxCACIS3-

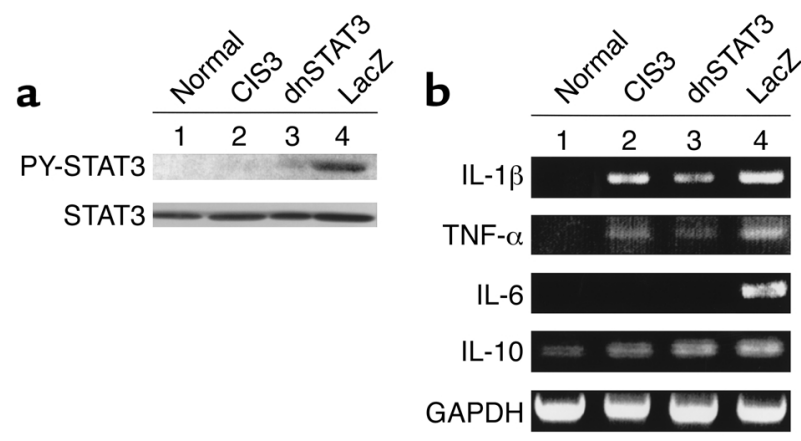

Figure 4

Effect of CIS3 and dnSTAT3 on STAT3 activation (a) and cytokine production (b) in the AIA model. CIS3, dnSTAT3, or LacZ viruses were infected on day 6 after the initial mBSA immunization. Ankle joints were removed and homogenized 28 days after immunization. The ankle joint of an untreated mouse was used as a normal control (normal). Cell extracts were immunoblotted with anti-phosphorylated STAT3 or anti-STAT3 Ab's. (b) Twenty-eight days after the first immunization, total RNA was extracted from ankle joints of mice infected with CIS3 (lane 2), dnSTAT3 (lane 3), or LacZ (lane 4) adenoviruses at day 6 . The ankle joint of an untreated mouse was used as a normal control (lane 1 ). RT-PCR analysis was performed to detect TNF- $\alpha$, IL-1 $\beta$, IL- 6 , and IL-10 levels. 


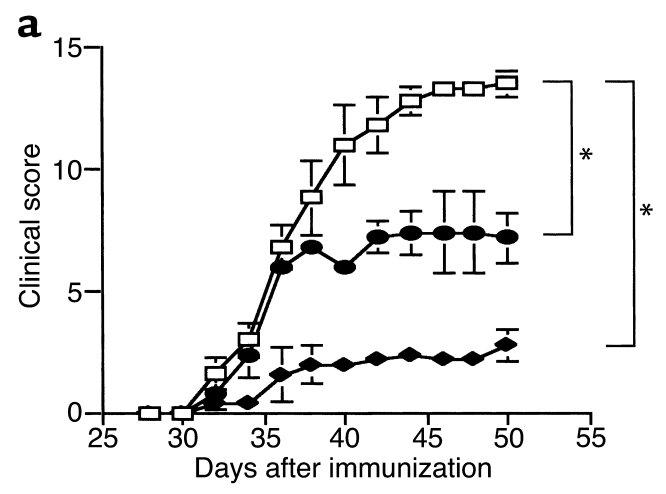

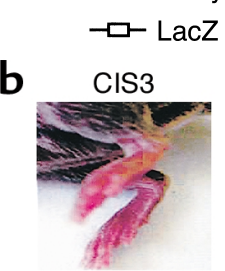

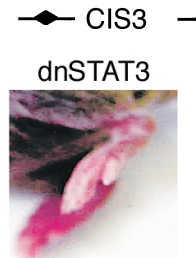

C CIS3

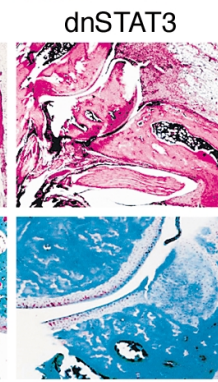

- dnSTAT3

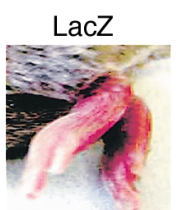

$\mathrm{H}-\mathrm{E}$

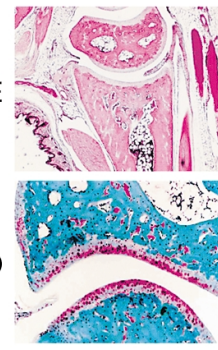

its
Safranin $\mathrm{O}$

\section{Figure 5}

Therapeutic effects of CIS3 virus injection in the CIA model. DBA/1J mice were immunized by intradermal injection at the base of the tail with $100 \mu \mathrm{g}$ of bovine type II collagen with Freund's complete adjuvant (day 0 ). On day 21 , mice received a booster dose of intradermal injection. On day 28, LPS was injected intraperitoneally, and adenoviruses were injected into ankle joints and wrists. (a) Severity of disease was assessed every other day using an established macroscopic scoring system ranging from 0 to 4 for each leg. Data represent mean clinical score of five mice from each group. (b) Macroscopic observations of the ankles of mice treated with LacZ, CIS3, or dnSTAT3 viruses on day $50 . \ln (\mathbf{c})$, ankle joint tissues were stained with $\mathrm{H}-\mathrm{E}$ and Safranin $O(\times 100) .{ }^{*} P<0.01$.

infected group, compared with the AxCALacZ-treated group. In contrast, local periarticular gene transfer of dnSTAT3 was also effective, but did not completely reduce the severity of arthritis, paw thickness, and redness compared with CIS3 adenovirus (Figure 5, a-c). We graded synovial tissue thickening, mononuclear cell infiltration, and cartilage and bone destruction by the pannus invasion with histopathological examination (Figure 5c). Each mouse exhibited much milder pathology in the AxCACIS3-treated joint than in the AxCALacZ-treated joint. The joints of AxCACIS3-treated mice exhibited much less inflammatory joint tissue and a strong reduction in bone erosion and number of inflammatory cells (Figure 5c). AxCAdnSTAT3 also reduced the pathological destruction of cartilage and bone, but it was not as effective as AxCACIS3. Measure- ment of cartilage thickness showed that the cartilage was well preserved in the AxCACIS3-treated joints. Comparison of histological grades and cartilage thickness between the two groups revealed that the differences were statistically significant $(P<0.01)$.

We next investigated whether the adenovirus was also effective after establishment of the disease. Adenoviruses were administered by periarticular injection into the wrist and ankle joints of mice 4 days after LPS injection (Figure 6). CIS3 adenovirus strongly blocked the progression of disease for 20 days after virus injection as assessed by clinical examination, paw thickness, and redness. Histological examination also confirmed the therapeutic effect of CIS3 adenovirus (data not shown). In contrast, the effect of dnSTAT3 adenovirus was transient (Figure 6); it suppressed development of arthritis for 10 days, but symptoms of arthritis reappeared thereafter. These results suggest that local CIS3 expression after gene transfer to joints with established disease could effectively protect tissue from inflammation and block bone erosion.

\section{Discussion}

The goal of this study was to extend our understanding of the roles played by STAT3 and a cytokine-signaling negative regulator, CIS3, in the pathogenesis of different arthritic diseases by interfering with cytokinesignaling pathways. Our study also aimed to develop a new therapeutic strategy for the treatment of RA by blockade of intracellular cytokine-signaling pathways.

We reported previously hyperactivation of STAT3 and CIS3 induction in inflammatory bowel diseases (IBD) and in mouse models of IBD (17). We demonstrated that IL-6 plays a crucial role in the development of colitis and that STAT3 activation is involved in the perpetuation of colitis. We also found that CIS3 was induced with the development of colitis, whereas in contrast, high-level CIS3 expression suppressed STAT3 activation. Forced expression of a dominant-negative form of CIS3 in mice resulted in more severe colitis. Thus, we

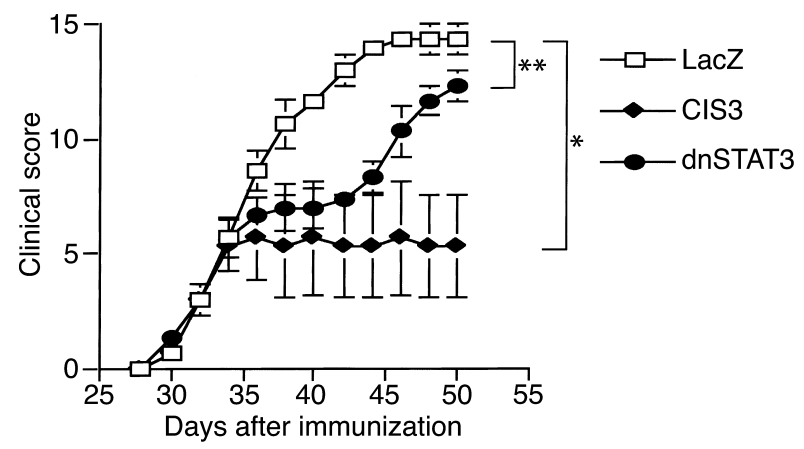

\section{Figure 6}

Effect of CIS3 adenovirus on established arthritis. Viruses were administered by periarticular injection into the wrist and ankle joints of mice 4 days after LPS injection. Severity of disease was assessed every other day ranging from 0 to 4 for each leg. Data represent mean clinical score of five mice from each group. ${ }^{*} P<0.01,{ }^{*} P<0.05$. 
proposed that CIS3 expression negatively regulates the pathogenesis of inflammation by modulating STAT3 activity in colitis. Similarly, we observed hyperactivation of STAT3 and CIS3 expression in RA patients and animal models of RA. Therefore, we propose here that STAT3 hyperactivation is related to the progression and/or development of RA, and CIS3 induction suppresses inflammation by reducing STAT3 activity.

Since CIS3 expression was induced subsequent to STAT3 activation (Figure 1, b and c), CIS3 probably acts as a negative-feedback regulator of STAT3. It is possible that CIS3 expression is one, if not the only, mechanism that negatively regulates the inflammatory reaction in arthritis. Consistent with this notion, expression of CIS3 by adenovirus before inflammation starts strongly suppressed AIA and CIA. However, we observed strong activation of STAT3 in RA tissues even in the presence of high CIS3 mRNA expression. This may be explained by modification or rapid degradation of CIS3 protein. We observed low levels of CIS3 protein in patients with Crohn disease and ulcerative colitis, although CIS3 mRNA expression was very high in the colon (17). As shown previously, CIS3 is very unstable and degraded by the ubiquitin/proteasome system (28). Furthermore, CIS3 is phosphorylated in response to cytokine stimulation (29). These modifications and the rapid degradation by proteasomes could be reasons underlying the incomplete suppression of STAT3 by CIS3 in chronic inflammation. Thus, we believe that additional expression of exogenous CIS3 by gene transfer is effective in suppressing both STAT3 and inflammation.

The severity of inflammatory reactions in ankle joints of AIA and CIA mice assessed by arthritis score and paw volume, significantly improved by local injection of CIS 3 and dnSTAT3 adenovirus. These in vivo effects of CIS3 and dnSTAT3 virus on inflammation and bone destruction in arthritic mice result from direct inhibition of synoviocytes and osteoclast activity by the virus. Since the efficiency of adenoviral gene transfer into lymphocytes is known to be very low, we conclude that the CIS3 virus suppressed the effector phase of arthritic bone destruction. However, we found that total IgG levels in ankle joints of CIA models were decreased strongly by CIS3 adenovirus and partially by dnSTAT3 virus (data not shown). Since these two viruses suppressed IL-6 production from synovial tissues, it is possible that these two viruses affect $\mathrm{T}$ cell differentiation and/or $\mathrm{Ab}$ production in B cells. Further study is necessary to clarify the effects of CIS3 and dnSTAT3 viruses on T or B cells.

As shown in Figures 5 and 6, the protective effect of dnSTAT3 against CIA was only partial compared with AIA, while CIS3 efficiently suppressed both (Figures 3 and 4). Both CIS3 and dnSTAT3 adenoviruses similarly suppressed STAT3 activation in the AIA model (Figure 4) and in RA synoviocytes (data not shown) at the same moi. Therefore, the superior effect of CIS3 on the CIA model may be due to the difference in the suppression mode of CIS3 and dnSTAT3 against cytokine signaling. We and others have shown that CIS3 can inhib- it many cytokine signaling pathways, including IFN- $\gamma$, IL-2, and IL-4, which activate STAT1, STAT5, and STAT6, respectively (11), while dnSTAT3 suppressed STAT3 pathway only. Furthermore, it has been shown that CIS3 inhibits all signals generated by JAKs, including the Ras-MAP kinase pathway (14). Therefore, a wide variety of signals including other STATs and MAP kinase generated by cytokines probably play an important role in the development of CIA (30), and CIS3 inhibits these signals. Furthermore, we found recently that CIS3, but not dnSTAT3, inhibits LPS-induced NO, TNF- $\alpha$, and IL-12 production in macrophages (ref. 13, and unpublished data). These data suggest that CIS3 expression in macrophages inhibits inflammatory reaction. In this context, CIS3 could inhibit inflammatory arthritis more efficiently than STAT3.

Although anti-TNF- $\alpha$ mAbs markedly ameliorate joint involvement in the majority of patients with RA (3, 4), blockade of IL-6, which is thought to be a downstream target of TNF- $\alpha$, would possibly be beneficial in the treatment of RA. Yoshizaki and coworkers (31) demonstrated the antiarthritic effect of humanized anti-human IL-6 receptor (IL-6R) Ab in CIA models using the cynomolgus monkey. Furthermore, administration of a mAb to IL- 6 in an open pilot trial of five patients with RA led to clinical and biological improvements (32). Our present study leads us to propose an alternative therapeutic strategy; blockade of intracellular cytokine-signaling pathways. Adenoviral gene transfer of cytokine signal inhibitors can efficiently suppress development of arthritis in CIA and AIA models. However, there are certain drawbacks in using adenovirus vectors for gene therapy of arthritis, since adenovirus vector-mediated gene transduction is transient, and local injection of adenovirus sometimes induces inflammation $(33,34)$. Thus, our study also raises another possible therapy using JAK1-specific inhibitors. Since gp130 mainly uses JAK1 (35), and JAK1 is not essential for hematopoiesis, with the exception of granulocyte development by G-CSF, JAK1 inhibitors may be useful therapeutic reagents for both RA and IBD. Our preliminary studies suggested that the JAK2 inhibitor, AG490 (36), was effective in the CIA model. We would anticipate better efficacy with a JAK1-specific inhibitor. An alternative approach of increasing CIS3 protein levels may also be possible for suppression of inflammation. We found that CIS3 protein levels were regulated not only by cytokines, but also by many other factors, including proteasome degradation, phosphorylation, cAMP levels, and cell differentiation. A reagent that stabilizes CIS3 protein or upregulates CIS3 mRNA levels may effectively suppress inflammation.

In summary, intervention of intracellular signal transduction pathways in synoviocytes by adenovirus-mediated gene transfer of CIS3 resulted in suppression of bone destruction and amelioration of inflammation in mouse autoimmune arthritis. Our results suggest that signaling mediated by JAK/STAT3 plays critical roles in the inflammation and joint destruction in RA. Aden- 
ovirus vectors are useful for in vivo gene transfer to intraarticular tissues, and adenovirus-mediated gene transfer of the CIS3 gene is a promising means of preventing arthritic bone destruction. No definitive cure for RA will be possible until its etiology is elucidated, however suppression of synoviocytes and osteoclast activity by gene transfer represents a novel therapeutic strategy for preventing the joint destruction associated with RA.

\section{Acknowledgments}

We thank H. Ohgusu, M. Sasaki, and Y. Kawabata for their excellent technical assistance and K. Yoshida for tissue collection. This work was supported in part by grants from the Ministry of Science, Education, Sports, and Culture of Japan; the Japan Health Science Foundation; the Human Frontier Science Program; and the Japan Research Foundation for Clinical Pharmacology.

1. Feldmann, M., Brennan, F.M., and Maini, R.N. 1996. Role of cytokines in rheumatoid arthritis. Annu. Rev. Immunol. 14:397-440.

2. Feldmann, M., Brennan, F.M., and Maini, R.N. 1996. Rheumatoid arthritis. Cell. 85:307-310.

3. Elliott, M.J., et al. 1994. Randomised double-blind comparison of chimeric monoclonal antibody to tumor necrosis factor alpha (cA2) versus placebo in rheumatoid arthritis. Lancet. 344:1105-1110.

4. Elliott, M.J., et al. 1994. Repeated therapy with monoclonal antibody to tumor necrosis factor alpha (cA2) in patients with rheumatoid arthritis. Lancet. 344:1125-1127.

5. Mihara, M., Moriya, Y., Kishimoto, T., and Ohsugi, Y. 1995. Interleukin6 (IL-6) induces the proliferation of synovial fibroblastic cells in the presence of soluble IL-6 receptor. Br. J. Rheumatol. 34:321-325.

6. Tamura, T., et al. 1993. Soluble interleukin- 6 receptor triggers osteoclast formation by interleukin 6. Proc. Natl. Acad. Sci. USA. 90:11924-11928.

7. Boe, A., Baiocchi, M., Carbonatto, M., Papoian, R., and Serlupi-Crescenzi, O. 1999. Interleukin 6 knock-out mice are resistant to antigeninduced experimental arthritis. Cytokine. 11:1057-1064.

8. Ohshima, S., et al. 1998. Interleukin 6 plays a key role in the development of antigen-induced arthritis. Proc. Natl. Acad. Sci. USA. 95:8222-8226.

9. Alonzi, T., et al. 1998. Interleukin 6 is required for the development of collagen-induced arthritis. J. Exp. Med. 187:461-468.

10. Yoshizaki, K., Nishimoto, N., Mihara, M., and Kishimoto, T. 1998. Therapy of rheumatoid arthritis by blocking IL- 6 signal transduction with a humanized anti-IL-6 receptor antibody. Springer Semin. Immunopathol. 20:247-259.

11. Yasukawa, H., Sasaki, A., and Yoshimura, A. 2000. Negative regulation of cytokine signaling pathway. Annu. Rev. Immunol. 18:143-164.

12. Bode, J.G., et al. 1999. LPS and TNFalpha induce SOCS3 mRNA and inhibit IL-6-induced activation of STAT3 in macrophages. FEBS Lett. 463:365-370.

13. Cassatella, M.A., et al. 1999. Interleukin-10 (IL-10) selectively enhances CIS3/SOCS3 mRNA expression in human neutrophils: evidence for an IL-10-induced pathway that is independent of STAT protein activation. Blood. 94:2880-2889.

14. Sasaki, A., et al. 1999. Cytokine-inducible SH2 protein-3 (CIS3/SOCS3) inhibits Janus tyrosine kinase by binding through the N-terminal kinase inhibitory region as well as SH2 domain. Genes Cells. 4:339-351.

15. Schmitz, J., Weissenbach, M., Haan, S., Heinrich, P.C., and Schaper, F.
2000. SOCS3 exerts its inhibitory function on interleukin- 6 signal transduction through the SHP2 recruitment site of gp130. J. Biol. Chem. 275:12848-12856.

16. Nicholson, S.E., et al. 2000. Suppressor of cytokine signaling-3 preferentially binds to the SHP-2-binding site on the shared cytokine receptor subunit gp130. Proc. Natl. Acad. Sci. USA. 97:6493-6498.

17. Suzuki, A., et al. 2001. CIS3/SOCS3/SSI3 plays a negative regulatory role in STAT3 activation and intestinal inflammation. J. Exp. Med. 193:471-481.

18. Nakajima, K., et al. 1996. A central role for STAT3 In IL-6-induced regulation of growth and differentiation in M1 leukemia cells. EMBOJ. 15:3651-3658.

19. Arnett, F.C., et al. 1988. The American Rheumatism Association 1987 revised criteria for the classification of rheumatoid arthritis. Arthritis. Rheum. 31:315-324.

20. Miyake, S., et al. 1996. Efficient generation of recombinant adenoviruses using adenovirus DNA-terminal protein complex and a cosmid bearing the full-length virus genome. Proc. Natl. Acad. Sci. USA. 93:1320-1324.

21. Taniguchi, K., et al. 1999. Induction of the p16INK4a senescence gene as a new therapeutic strategy for the treatment of rheumatoid arthritis. Nat. Med. 5:760-767.

22. Takayanagi, H., et al. 1999. Suppression of arthritic bone destruction by Adenovirus-mediated csk gene transfer to synoviocytes and osteoclast. J. Clin. Invest. 104:137-146.

23. Brackertz, D., Mitchell, G.F., and Mackay, I.R. 1977. Antigen-induced arthritis in mice. Arthritis Rheum. 20:841-850.

24. Holmdahl, R., et al. 1989. Collagen induced arthritis as an experimental model for rheumatoid arthritis. Immunogenetics, pathogenesis and autoimmunity. APMIS. 97:575-584.

25. Zhang, D., et al. 1998. Restraint stress-induced immunosuppression by inhibiting leukocyte migration and Th1 cytokine expression during the intraperitoneal infection of Listeria monocytogenes. J. Neuroimmunol. 92:139-151.

26. Kim, S.H., et al. 2000. Gene therapy for established murine collageninduced arthritis by local and systemic adenovirus-mediated delivery of interleukin-4. Arthritis Res. 2:293-302.

27. Gerlag, D.M., et al. 2000. The effect of a T cell-specific NF- $\kappa B$ inhibitor on in vitro cytokine production and collagen-induced arthritis. $J$. Immunol. 165:1652-1658.

28. Kamura, T., et al. 1998. The Elongin BC complex interacts with the conserved SOCS-box motif present in members of the SOCS, ras, WD-40 repeat, and ankyrin repeat families. Genes Dev. 12:3872-3881.

29. Sasaki, A., et al. 2000. CIS3/SOCS3 suppresses erythropoietin signaling by binding the EPO receptor and JAK2. J. Biol. Chem. 275:29338-29347.

30. Muller-Ladner, U., et al. 2000. Activation of the IL-4 STAT pathway in rheumatoid synovium. J. Immunol. 164:3894-3901.

31. Mihara, M., et al. 2001. Humanized antibody to human interleukin-6 receptor inhibits the development of collagen arthritis in cynomolugus monkeys. Clin. Immunol. 98:319-326.

32. Wendling, D., Racadot, E., and Wijdenes, J. 1993. Treatment of severe rheumatoid arthritis by anti-interleukin 6 monoclonal antibody. $J$. Rheumatol. 20:259-262.

33. Varley, A.W., Coulthard, M.G., Meidell, R.S., Gerard, R.D., and Munford, R.S. 1995. Inflammation-induced recombinant protein expression in vivo using promoters from acute-phase protein genes. Proc. Natl. Acad. Sci. USA. 92:5346-5350.

34. Byrnes, A.P., Rusby, J.E., Wood, M.J., and Charlton, H.M. 1995. Adenovirus gene transfer causes inflammation in the brain. Neuroscience. 66:1015-1024.

35. Guschin, D., et al. 1995. A major role for the protein tyrosine kinase JAK1 in the JAK/STAT signal transduction pathway in response to interleukin-6. EMBO. J. 14:1421-1429.

36. Meydan, N., et al. 1996. Inhibition of acute lymphoblastic leukemia by a Jak-2 inhibitor. Nature. 379:645-648. 